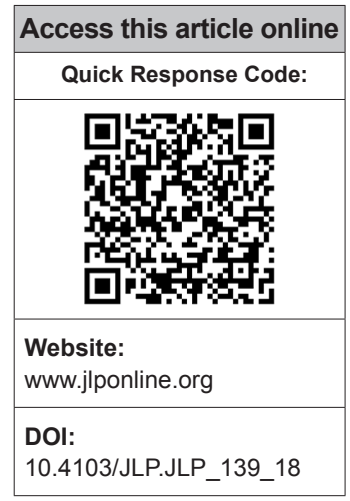

Departments of Lab Medicine and ${ }^{1}$ Orthopaedics, JPNA Trauma Centre, All India Institute of Medical Sciences, New Delhi, India

Address for correspondence:

Dr. Purva Mathur,

Room No. 211,

$2^{\text {nd }}$ Floor, Department of Laboratory Medicine,

JPNA Trauma Centre, AllMS, New Delhi, India.

E-mail: purvamathur@ yahoo.co.in

Submission: 15-10-2018 Accepted: 22-03-2019

\title{
Antibiotic resistance profile and co-production of extended spectrum beta lactamases and AmpC in Acinetobacter spp. in a level 1 trauma center from India
}

\author{
Priyam Batra, Surbhi Khurana, Aishwarya Govindaswamy, Anjana Aravinda, \\ Vijeta Bajpai, Muruganantham Ayyanar, Purva Mathur, Rajesh Malhotra ${ }^{1}$
}

\begin{abstract}
:
INTRODUCTION: Acinetobacter baumannii has now emerged as a significant nosocomial pathogen in health-care setting ESP in intensive care units. Rapidly growing resistance among clinical isolates suggests a need to detect resistance mechanisms in this organism. The present study was designed to compare the various phenotypic tests available with the gold standard of genotype.

METHODOLOGY: The present study was conducted to include all isolates of Acinetobacter spp. isolated over 3 years. Their resistance to various antibiotics was determined and extended spectrum beta-lactamases (ESBL) and AmpC production in the isolates showing resistance to ceftazidime/ ceftriaxone/cefotaxime (CAZ/CTR/CTX) was determined. ESBL and AmpC production was confirmed using polymerase chain reaction (PCR).
\end{abstract}

RESULTS: A total of 154 strains were isolated, and all the strains were tested for ESBL and AmpC detection. Of the strains tested, 15 (9.7\%), 17 (11\%), 24 (15.6\%), 27 (17.5\%), $54(35 \%), 67(43.5 \%)$, and $72(46.7 \%)$ strains showed ESBL production using CTX/CTX-clavulanate double-disc synergy test (DDST), CTX/CTX-clavulanate E-test, CAZ/CAZ-clavulanate DDST, CAZ/CAZ-clavulanate E-test, Piperacillin/Piperacillin-tazobactam (TZ) DDST, CTR/CTR-Sulbactum DDST, and Piperacillin/Piperacillin-TZ E-test, respectively. 20 (12.9\%) and 19 (12.3\%) of strains were positive for AmpC production using AmpC disc test and Boronic acid inhibition test, respectively. Genotype analysis using PCR for TEM, SHV, CTXM, PER, and VEB genes was done and 69 (51.5\%) strains were positive for TEM gene.

DISCUSSION: ESBL detection in Acinetobacterspp. is difficult as standard guidelines for the same are not available unlike in enterobacteriaceae, and there are no zone diameter breakpoints for aztreonam and cefpodoxime. In comparison, piperacillin/piperacillin-TZ E-test had the best sensitivity and specificity for ESBL detection.

CONCLUSION: Standard guidelines for ESBL detection in nil fermeners like Acinetobacter spp. must be laid down for ease of detection. Use of piperacillin/piperacillin-tazobactam E-test could be used as one of the standard methods.

Key words:

Acinetobacter spp., AmpC, antibiotic resistance, extended spectrum beta-lactamases

\section{Introduction}

A cinetobacter baumannii, named after

This is an open access journal, and articles are distributed under the terms of the Creative Commons Attribution-NonCommercial-ShareAlike 4.0 License, which allows others to remix, tweak, and build upon the work non-commercially, as long as appropriate credit is given and the new creations are licensed under the identical terms.

For reprints contact: reprints@medknow.com and water and was earlier considered to be a normal commensal of the human flora. ${ }^{[1]}$ However, it has now emerged as the most significant nosocomial pathogen in the

How to cite this article: Batra $P$, Khurana $S$, Govindaswamy A, Aravinda A, Bajpai V, Ayyanar M, Mathur P, Malhotra R. Antibiotic resistance profile and co-production of extended spectrum beta lactamases and AmpC in Acinetobacter spp. in a level 1 trauma center from India. J Lab Physicians 2019;11:128-32. 
health-care setting ESP in intensive care units (ICUs) ${ }^{[2]}$ due to its virulence properties and its ability to acquire resistance to various antibiotics. ${ }^{[3]}$ Reports of community-acquired Acinetobacter infections have increased over the past decade. ${ }^{[4]}$ Acinetobacter spp. have been associated with a wide range of infections such as pneumonia, bloodstream infection, surgical site infection, and urinary tract infection. ${ }^{[5]}$ The mortality of patients with $A$. baumannii infections in hospitals and in the ICU has ranged from $7.8 \%$ to $23 \%$ and from $10 \%$ to $43 \%$, respectively. ${ }^{[6]}$

The extent of antimicrobial resistance is more severe in A. baumannii isolates from patients in Asian and European ICUs than from patients in American ICUs. ${ }^{[7]}$ A significant increase in antimicrobial resistance has been noted worldwide from 2004 to 2009. ${ }^{[6]}$ Acinetobacter spp. have a number of resistance mechanisms such as $\beta$ lactamase enzyme production, aminoglycoside-modifying enzyme, porin mutation, and efflux pumps which confer resistance to large number of antibiotics. The first MDRAB isolate resistant to almost all available antibiotics in Taiwan was discovered in $1998 .{ }^{[8]}$ A high level of carbapenem-resistant Acinetobacter spp. have also been reported from the Indian ICUs with the rate of resistance ranging from $76 \%$ to $90 \% .[9,10]$ The spread of multidrug-resistance determinants in A. baumannii is mostly through plasmid conjugation, transposon acquisition or integron mobilization to gain clusters of genes encoding resistance to several antibiotic families. ${ }^{[11]}$

Extended spectrum beta-lactamases (ESBLs) in $A$. baumannii are widely reported from many countries, such as India, France, Turkey, Korea, Belgium, Latin America, the United Kingdom, and the United States. ${ }^{[2]}$ Acinetobacter inherently produces chromosomally mediated AmpC type cephalosporinases which are also known as Acinetobacter-derived cephalosporinases. ${ }^{[12]}$ Co-production of ESBLs and AmpC $\beta$-lactamases is a major problem which is responsible for causing therapeutic failures with the use of most of the antibiotics. Rapidly growing resistance among clinical isolates suggests a need to detect resistance mechanisms in this organism. Various methods are available for detection of ESBL and AmpC production. However, none of these tests has been found to be the best in Acinetobacter spp. ${ }^{[13]}$ Thus, the present study was designed to compare the various phenotypic tests of ESBL and AmpC production available with the gold standard of genotype.

\section{Methodology}

The study was conducted over a period of 3 years (2013-2015) at a 186 bedded level 1 trauma center of
India. All consecutive strains of Acinetobacter spp. isolated from the various clinical samples were included in the study. All the strains isolated were identified using the Vitek 2 system, Biomerieux, and France (GNID ref 21341) and their antibiotic sensitivity was done using the disc diffusion method as per the CLSI guidelines. The antibiotics tested were: ceftazidime (CAZ) $(30 \mu \mathrm{g})$, cefotaxime $(\mathrm{CTX})(30 \mu \mathrm{g})$, ceftriaxone (CTR) $(30 \mu \mathrm{g})$, cefepime (CPM) $(30 \mu \mathrm{g}), \mathrm{CPM} /$ tazobactam (CPM/TZ) (30/10 $\mu \mathrm{g})$, cefoperazone/sulbactam (SUL), imipenem (IMI) $(10 \mu \mathrm{g})$, meropenem (MERO) $(10 \mu \mathrm{g})$, amikacin $(30 \mu \mathrm{g})$, netilmicin $(30 \mu \mathrm{g})$, levofloxacin $(5 \mu \mathrm{g})$, tigecycline (TGC) $(15 \mu \mathrm{g})$, and colistin (CL) $(10 \mu \mathrm{g})$.

All isolates found resistant to either CTX, CAZ, or CTR were tested for ESBL and AmpC production. ESBL detection was done using a double-disc synergy test (DDST) and E-test for ESBL detection using clavulanic acid or $\mathrm{TZ}$ as an inhibitor. AmpC detection was done using AmpC disc test and boronic acid inhibition test.

\section{Extended spectrum beta-lactamases detection} Double-disc synergy test ${ }^{[13]}$

The test was performed using ceftazidime/ CAZ-clavulanate, CTX/CTX-clavulanate, piperacillin/ piperacillin-TZ, and CTR/CTR-SUL discs. A $\geq 5 \mathrm{~mm}$ increase in the zone size was considered as positive for ESBL production.

E-test for extended-spectrum beta-lactamases detection ${ }^{[13]}$ Minimum inhibitory concentrations (MICs) of CTX and CAZ with and without clavulanic acid; and piperacillin/ piperacillin-TZ were tested by the E-test method as per the manufacturer's instructions. An $\geq 8$-fold reduction in cephalosporins/piperacillin MICs in the presence of clavulanate/TZ was taken as confirmatory of ESBL.

\section{AmpC detection}

AmpC disc test ${ }^{[14]}$

A lawn culture of Escherichia coli ATCC 25922 was grown on a Mueller-Hinton Agar plate. Several colonies of test organism were inoculated on sterile discs $(6 \mathrm{~mm})$ which were moistened with sterile saline $(20 \mu \mathrm{l})$. The inoculated disc was placed beside a cefoxitin disc on agar plate. The plates were incubated overnight at $35^{\circ} \mathrm{C}$. A positive test was considered to be either flattening or indentation of the cefoxitin inhibition zone, which indicated enzymatic inhibition of cefoxitin. An undistorted zone was suggestive of a negative test. ${ }^{[14]}$

\section{Boronic acid disk test method}

For this, the $30 \mu \mathrm{g}$ cefoxitin disk will be supplemented with $300 \mu \mathrm{g}$ of phenylboronic acid. An organism demonstrated a defined increase $(\geq 5 \mathrm{~mm})$ in zone diameter around the antibiotic disk with added 
inhibitor compound compared to that with the antibiotic-containing disk alone will be considered to be an AmpC producer..$^{[15]}$

\section{Genotype analysis}

DNA was extracted using the standard method, and PCR for ESBL and AmpC production was performed.

Polymerase chain reaction for extended spectrum beta-lactamases detection ${ }^{[16]}$

Suspected/phenotypically confirmed ESBL producing strains were examined for the presence of the $b l a_{\mathrm{TEM}^{\prime}}$ $b l a_{\mathrm{SHV}}, b l a_{\mathrm{CTX}-\mathrm{M}^{\prime}} b l a_{\mathrm{PER}^{\prime}}$ and $b l a_{\mathrm{VEB}} \beta$-lactamases genes, using the primers listed in Table 1.

Polymerase chain reaction protocol for AmpC production $^{[16]}$

Multiplex PCR was performed for the detection of MOX-1, MOX-2, CMY-1, CMY-8 TO CMY-11, LAT-1 TO LAT-4, CMY-2 to CMY-7, BIL-1, DHA-1, DHA-2, ACC, MIR-1T ACT-1, FOX-1 to FOX-5B. The primers used for PCR amplification are listed in Table 2.

\section{Ethical clearance}

This study (project code: I-800, grant number: 5/3/3/26/2011-ECD-I) was approved by the Institute Ethics Committee, All India Institute of Medical Sciences, New Delhi.

\section{Statistical analysis}

Data were analyzed statistically using the SPSS software version 6.0. (SPSS Inc., Chicago, Illinois, USA).

\section{Results}

A total of 154 strains of Acinetobacter spp. were isolated and identified. The antibiotic resistance pattern of the strains isolated is shown in Figure 1.

Of the 154 strains tested, only 8 (5.1\%), $2(1.2 \%)$, and $3(1.9 \%)$ strains, respectively, were sensitive to CAZ, CTX, and CTR. Thus, all the strains were tested for ESBL and AmpC production. Of the strains tested, $15(9.7 \%), 17(11 \%), 24(15.6 \%), 27$ (17.5\%), 54 (35\%), $67(43.5 \%)$, and $72(46.7 \%)$ strains showed ESBL production using CTX/CTX-clavulanate DDST, CTX/CTX-clavulanate E-test, CAZ/CAZ-clavulanate DDST, CAZ/CAZ-clavulanate E-test, Piperacillin/ Piperacillin-TZ DDST, CTR/CTR-Sulbactum DDST, and Piperacillin/Piperacillin-TZ E-test, respectively. $20(12.9 \%)$ and $19(12.3 \%)$ of strains were positive for AmpC production using AmpC disc test and boronic acid inhibition test, respectively.

Genotype analysis using PCR for TEM, SHV, CTXM, PER, and VEB genes showed that 69 (51.5\%), 25 (21.1\%),

Table 1: Primer and polymerase chain reaction conditions for amplification of $\beta$-lactamases

\begin{tabular}{lllc}
\hline Primer name & Sequence & PCR conditions & Amplicon size (bp) \\
\hline TEM-F & AGATCAGTTGGGTGCACGAG & $1.5 \mathrm{mMMg} ; 55^{\circ} \mathrm{C}$ annealing & 750 \\
TEM-R & TGCTTAATCAGTGAGGCACC & & \\
SHV-F & GGGAAACGGAACTGAATGAG & $1.5 \mathrm{mMMg} ; 55^{\circ} \mathrm{C}$ annealing & 380 \\
SHV-R & TTAGCGTTGCCAGTGCTCG & & \\
CTX-MU-1 & ATGTGCAGCACCAGTAACGT & $1.5 \mathrm{mM} \mathrm{Mg} ; 58^{\circ} \mathrm{C}$ annealing, $1 \mathrm{~min}$ & \\
CTX-MU-2 & TGGGTCAACTAGGTGACCAGA & & \\
PER-F & ATGAATGTCATTATAAAAGC & $1.5 \mathrm{mM} \mathrm{Mg} ; 55^{\circ} \mathrm{C}$ annealing \\
PER-R & AATTTGGGCTTAGGGCAGAA & & \\
VEB-F & GTTAGCGGTAATTTAACCAG & $1.5 \mathrm{mM} \mathrm{Mg} ; 55^{\circ} \mathrm{C}$ annealing \\
VEB-R & TATTCAAATAGTAATTCCACG & & 826 \\
\hline PCR & &
\end{tabular}

$\mathrm{PCR}=$ Polymerase chain reaction

Table 2: Primers used for amplification of AmpC genes

\begin{tabular}{|c|c|c|c|}
\hline Target (s) & Primer & Sequence (5' to $\left.3^{\prime}\right)$ & Expected amplicon size (bp) \\
\hline \multirow[t]{2}{*}{ MOX-1, MOX-2, CMY-1, CMY-8 TO CMY-11 } & MOXMF & GCT GCT CAA GGA GCA CAG GAT & 520 \\
\hline & MOXMAR & CAC ATT GAC ATA GGT GTG GTG C & \\
\hline \multirow[t]{2}{*}{ LAT-1 TO LAT-4, CMY-2 to CMY-7, BIL-1 } & CITMF & TGG CCA GAA CTG ACA GGC AAA & 462 \\
\hline & CITMR & TTT CTC CTG AAC GTG GCT GGC & \\
\hline \multirow[t]{4}{*}{ DHA-1, DHA-2 } & DHAMF & AAC TTT CAC AGG TGT GCT GGG T & 405 \\
\hline & DHAMR & CCG TAC GCA TAC TGG CTT TGC & \\
\hline & ACCMF & AAC AGC CTC AGC AGC CGG TTA & 346 \\
\hline & ACCMR & TTC GCC GCA ATC ATC CCT AGC & \\
\hline \multirow[t]{2}{*}{ MIR-1T ACT-1 } & EBCMF & TCG GTA AAG CCG ATG TTG CGG & 302 \\
\hline & EBCMR & CTT CCA CTG CGG CTG CCA GTT & \\
\hline \multirow[t]{2}{*}{ FOX-1 TO FOX-5B } & FOXMF & AAC ATG GGG TAT CAG GGA GAT G & 190 \\
\hline & FOXMR & CAA AGC GCG TAA CCG GAT TGG & \\
\hline
\end{tabular}


$40(30.3 \%), 50(44.2 \%)$, and $3(4.3 \%)$ strains, respectively, were positive for TEM, SHV, CTXM, PER, and VEB genes. PCR for AmpC genes showed that only 24 (9.4\%) strains produced DHAM gene. None of the strains showed production of MOXM, CITM, ACCM, EBCM, and FOXM. Using the SPSS software sensitivity and specificity of the tests was found which is given in Table 3. ESBL and AmpC co-production was seen in $18(11.7 \%)$ strains.

\section{Discussion}

MDR Acinetobacter spp. have been isolated worldwide. The rate of carbapenem resistance in Acinetobacter spp. isolated from Europe has been increasing with the lowest rates being reported from Scandinavia. ${ }^{[17]}$ According to MYSTIC surveillance study conducted in the year $2002-004$ in 48 European hospitals, $26.9 \%$, 30.2\%, 67.6\%, $66 \%$, and $52.4 \%$ of the strains were resistant to MERO, IMI, CAZ, ciprofloxacin, and gentamicin respectively. ${ }^{[18]}$ The prevalence of carbapenem-resistant Acinetobacter spp. from a burns unit in the USA was found to be substantially high, being $87 \%$ in the year $2007 .{ }^{[19]}$ In the Asian subcontinent, the level of resistance to all the available antibiotics including TGC and CL is very high. ${ }^{[20]}$ The level of CL resistance has been variable but is ever increasing ranging from; $2.7 \%$ from Europe ${ }^{[20]} 2 \%$ in the UK, ${ }^{[21]}$ and only sporadic cases from Slovakia. ${ }^{[22]}$ Similarly, in our study also very high resistance was seen to all the available antibiotics.

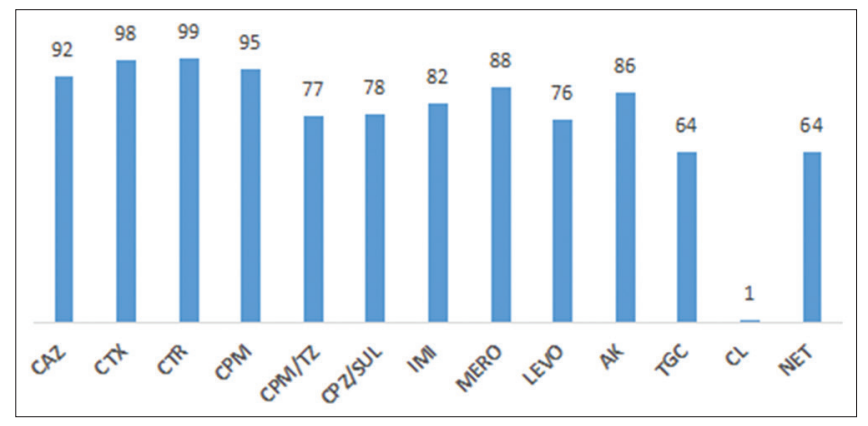

Figure 1: Antibiotic resistance profile (\% resistance) of Acinetobacter spp.
ESBL detection in Acinetobacter spp. is difficult as standard guidelines for the same are not available unlike in Enterobacteraceae. Unlike Enterobacteriaceae, there are no zone diameter breakpoints for aztreonam and cefpodoxime in Acinetobacter spp. ${ }^{[23]}$ Thus, in the present study, isolates showing resistance to CAZ, CTR, and CTX were tested for ESBL detection. ESBL detection was assessed using TZ, sulbactam, and clavulanate as inhibitors and was compared with PCR (taking PCR as the gold standard). In comparison, tests performed using SUL and TZ as inhibitors performed better than tests with clavulanic acid inhibitor. AmpC producing organisms act as hidden reservoirs for ESBLs. Such isolates, when tested using clavulanic acid inhibition test, are induced to produce high levels of AmpC enzymes which may antagonize the synergy arising from inhibition of ESBLsleading to false negative results. SUL and TZ are much less likely to induce AmpC $\beta$-lactamases and therefore, are preferable inhibitors for ESBL detection tests. ${ }^{[12]}$ However, few studies using clavulanic acid as inhibitor for DDST have reported up to 28\% ESBL production in Acinetobacter spp. ${ }^{[24]}$

The prevalence of AmpC $\beta$-lactamase producing Acinetobacter spp. appears to be increasing and they have been associated with increased nosocomial infections. However, in the present study, only $12 \%$ of strains showed AmpC $\beta$-lactamase production. Other authors also detected AmpC $\beta$-lactamases in $6(50 \%)$ out of 12 isolates of the Acinetobacter species using the same method. ${ }^{[25]}$

Genotypically, TEM gene was found the most common gene in our hospital isolates. Safari et al. from Iran reported SHV gene as the most frequent gene in 58\% of the strains. ${ }^{[26]}$ Pragasam et al. from India reported TEM (54\%), SHV (16\%), and PER (1\%) to be the most abundant in strains isolated from South India. ${ }^{[27]}$

Most of the ESBL producing strains in our study were resistant to almost all the antibiotics. This could be due to the expression of other resistance mechanisms such as production of carbapenemases, porin mutation, and outer membrane impermeability, The presence of resistance

Table 3: Statistical analysis of the tests

\begin{tabular}{|c|c|c|c|c|}
\hline Test & Sensitivity (\%) & Specificity (\%) & Positive predictive value (\%) & Negative predictive value (\%) \\
\hline CAZ/Cefta-clav DDST & 25.8 & 62.5 & 50 & 36.7 \\
\hline CAZ/Cefta-clav Etest & 29.03 & 65.62 & 55.1 & 38.9 \\
\hline CTX/Cefo-clav DDST & 16.13 & 56.25 & 34.8 & 31.58 \\
\hline CTX/Cefo-clav Etest & 18.28 & 57.8 & 38.6 & 32.7 \\
\hline Piperacillin/Pipera-Tazo DDST & 67.1 & 79.7 & 83.8 & 61.5 \\
\hline Piperacillin/Pipera-Tazo Etest & 82.42 & 87.3 & 90.4 & 77.5 \\
\hline CTR/Ceftria-Sulbactum DDST & 69 & 79.7 & 83.8 & 63 \\
\hline AmpC disc test & 83.3 & 86.1 & 52.6 & 96.6 \\
\hline Boronic acid inhibition test & 79.2 & 76.9 & 38.8 & 95.2 \\
\hline
\end{tabular}

CAZ=Ceftazidime, CTX=Cefotaxime, CTR=Ceftriaxone, DDST=Double-disc synergy test 
to beta-lactams is generally associated with resistance to other groups of antibiotics such as aminoglycosides and fluoroquinolones. Resistance mechanisms are generally transferable by way of plasmids. ${ }^{[2]}$ According to the growing and threatening danger of these bacteria, the rapid identification and detection of this strain have an important role in preventing their spread. Furthermore, these findings illuminate the necessity of review in antimicrobial therapy, use of proper antibiotics, and the utilization of new antimicrobial elements to treat the infections resulting from these bacteria. This is a big lacuna of the current study as it was not clinically correlated with the response of the patient to the antibiotics.

\section{Conclusion}

Standard guidelines for ESBL detection in nil fermeners like Acinetobacter spp. must be laid down for ease of detection. Use of piperacillin/piperacillin-tazobactam E-test could be used as one of the standard methods.

\section{Acknowledgment}

We would like to acknowledge the administration of JPNATC, AIIMS for providing the infrastructure to perform the tests.

\section{Financial support and sponsorship}

We would like to thank the Indian Council of Medical Research for providing the fund for conducting this study.

\section{Conflicts of interest}

There are no conflicts of interest.

\section{References}

1. Baumann P. Isolation of Acinetobacter from soil and water. J Bacteriol 1968;96:39-42.

2. Peleg AY, Seifert H, Paterson DL. Acinetobacter baumannii: Emergence of a successful pathogen. Clin Microbiol Rev 2008;21:538-82.

3. Fournier PE, Richet H. The epidemiology and control of Acinetobacter baumannii in health care facilities. Clin Infect Dis 2006;42:692-9.

4. Dijkshoorn L, Nemec A, Seifert H. An increasing threat in hospitals: Multidrug-resistant Acinetobacter baumannii. Nat Rev Microbiol 2007;5:939-51.

5. Maragakis LL, Perl TM. Acinetobacter baumannii: Epidemiology, antimicrobial resistance, and treatment options. Clin Infect Dis 2008;46:1254-63.

6. Falagas ME, Bliziotis IA, Siempos II. Attributable mortality of Acinetobacter baumannii infections in critically ill patients: A systematic review of matched cohort and case control studies. Crit Care 2006;10:R48.

7. Falagas ME, Karveli EA. The changing global epidemiology of Acinetobacter baumannii infections: A development with major public health implications. Clin Microbiol Infect 2007;13:117-9.

8. Hsueh PR, Teng LJ, Chen CY, Chen WH, Yu CJ, Ho SW, et al. Pandrug-resistant Acinetobacter baumannii causing nosocomial infections in a university hospital, Taiwan. Emerg Infect Dis 2002;8:827-32.
9. Sara A, Aroma M, Sheeba O, Kaur MP. Acinetobacter infections in a tertiary level intensive care unit in northern India: Epidemiology, clinical profiles and outcomes. J Infect Public Health 2012;5:145-52.

10. Sivaranjani V, Umadevi S, Srirangaraj S, Kali A, Seeth KS. Multi-drug resistant Acinetobacter species from various clinical samples in a tertiary care hospital from South India. Australas Med J 2013;6:697-700.

11. Esterly J, Richardson CL, Eltoukhy NS, Qi C, Scheetz MH. Genetic mechanisms of antimicrobial resistance of Acinetobacter baumannii. Ann Pharmacother 2011;45:218-28.

12. Singla P, Sikka R, Deeep A, Gagneja D, Chaudhary U. Co-production of ESBL and AmpC $\beta$-lactamases in clinical isolates of $A$. baumannii and A. lwoffii in a teaching tertiary care hospital. J Clin Diagn Res 2014;8:DC16-9.

13. Litake GM, Ghole VS, Niphadkar KB, Joshi SG. Phenotypic ESBL detection in Acinetobacter baumannii: A real challenge. Am J Infect Dis 2015;11:48.53.

14. Black JA, Moland ES, Thomson KS. AmpC disk test for detection of plasmid-mediated AmpC $\beta$-lactamases in Enterobacteriaceae lacking chromosomal AmpC $\beta$-lactamases. J Clin Microbiol 2005;43:3110-3.

15. Jacoby GA. AmpC $\beta$-lactamases. Clin Microbiol Rev 2009;22:161-82.

16. Khurana S, Mathur P, Kapil A, Valsan C, Behera B. Molecular epidemiology of beta-lactamase producing nosocomial gram-negative pathogens from North and South Indian hospitals. J Med Microbiol 2017;66:999-1004.

17. Kallman O, Lundberg C, Wretlind B, Ortqvist A. Gram negative bacteria from patients seeking medical advice in Stockholm after the tsunami catastrophe. Scand J Infect Dis 2006;38:448-50.

18. Unal S., Rodriguez JA. Activity of meropenem and comparators against Pseudomonas aeruginosa and Acinetobacter spp. Isolated in the MYSTIC program, 2002-2004. Diagn Microbiol Infect Dis 2005;53:265-71.

19. Trottier V, Segura PG, Namias N, King D, Pizano LR, Schulman CI. Outcomes of Acinetobacter baumannii infection in critically ill burned patients. J Burn Care Res 2007;28:248-54.

20. Gales AC, Jones RN, Sader HS. Global assessment of the antimicrobial activity of polymyxin B against 54,731 clinical isolates of gram-negative bacilli: Report from the SENTRY antimicrobial surveillance programme (2001-2004). Clin Microbiol Infect 2006;12:315-21.

21. Henwood CJ, Gatward T, Warner M, James D, Stockdale MW, Spence RP, et al. Antibiotic resistance among clinical isolates of Acinetobacter in the UK, and in vitro evaluation of tigecycline (GAR-936). J Antimicrob Chemother 2002;49:479-87.

22. Beno P, Krcmery V, Demitrovicova A. Bacteraemia in cancer patients caused by colistin-resistant gram-negative bacilli after previous exposure to ciprofloxacin and/or colistin. Clin Microbiol Infect 2006;12:497-8.

23. Clinical Laboratory Standards Institute. Performance Standards of Antimicrobial Susceptibility Testing; $26^{\text {th }}$ International Supplement CLSI Document M100-S25. Vol. 31. Clinical Laboratory Standards Institute; 2016.

24. Sinha M, Srinivasa H, Macaden R. Antibiotic resistance profile and amp; extended spectrum beta-lactamase (ESBL) production in Acinetobacter species. Indian J Med Res 2007;126:63-7.

25. Mohamudha Parveen R, Harish BN, Parija SC. Ampc beta lactamases among gram negative clinical isolates from a tertiary hospital, South India. Braz J Microbiol 2010;41:596-602.

26. Safari M, Mozaffari Nejad AS, Bahador A, Jafari R, Alikhani MY. Prevalence of ESBL and MBL encoding genes in Acinetobacter baumannii strains isolated from patients of intensive care units (ICU). Saudi J Biol Sci 2015;22:424-9.

27. Pragasam AK, Vijayakumar S, Bakthavatchalam YD, Kapil A, Das BK, Ray P, et al. Molecular characterisation of antimicrobial resistance in Pseudomonas aeruginosa and Acinetobacter baumannii during 2014 and 2015 collected across India. Indian J Med Microbiol 2016;34:433-41. 